\title{
Earnings Inequality in Brazil: Is it Permanent or Transitory?*
}

\author{
Antonio Loureiro Santos ${ }^{* *}$ \\ André Portela Souza ${ }^{* * *}$
}

\begin{abstract}
This paper seeks to analyze the dynamic behavior of prime-age male workers' earnings inequality in the formal labor market of the State of São Paulo in the years 1990-1998. The aim is to fit an econometric model of unobserved earnings components into the empirical earnings variance-covariance structure in order to evaluate the relative magnitude of individual characteristics and earnings instability as factors behind overall inequality. The analysis is based on a panel dataset constructed from RAIS data (official formal labor market data); several different models are estimated and tested using minimum distance techniques. Our results show that (i) the relative magnitude of the earnings instability component of inequality is much smaller than the individual characteristics component, and (ii) the observed heterogeneity, as accounted by education and age, explains only a little part of the inequality derived from individual characteristics.
\end{abstract}

Keywords: Earnings Inequality, Variance Components.

JEL Codes: J30, J60, O15.

\footnotetext{
${ }^{*}$ Submitted in February 2006. Revised in April 2007. The authors thank Naércio Menezes Filho, Paulo Picchetti, Sérgio Firpo and two anonymous referees for their comments. Antonio thanks FAPESP for the financial support.

${ }^{* *}$ IPE/FEA/USP. E-mail: antonio.a.santos@fazenda.gov.br

***EESP/FGV. E-mail: aps@fgvsp.br
}

Brazilian Review of Econometrics v. $27, \mathrm{n}^{\circ} 2$, pp. $259-283 \quad$ November 2007 


\section{Introduction}

Inequality and poverty are recurring issues in the Brazilian economic literature. Analysis typically involves the in-depth diagnosis of such problems in a given period of time; alternatively, many studies aim to compare the current state of the problem with its situation in a given period in the past. Certainly, such static and comparative statics analyses do provide us with a lot of insight into these issues; nevertheless, the time dynamic dimension of such issues has been comparatively underplayed.

The widespread use of panel data, on the other hand, has enabled researchers to use analytical approaches which focus mainly on the dynamic aspects of inequality. In this regard, the present work proposes a study of the dynamic structure of inequality based on a panel dataset constructed from RAIS data (official formal labor market data). The sample consists of prime-age male workers employed in the formal sector of the state of São Paulo, Brazil, in the 1990-1998 period. The autocovariance structure of earnings is decomposed into its permanent and transitory components, and the variance of earnings distribution is used as inequality measure.

In short, the proposed methodology departs from a permanent-transitory model of earnings components much like in the spirit of Friedman's permanent income model. From such a model, theoretical variances and autocovariances can be derived, which are then adjusted to their empirical counterparts. This methodology allows answering the following questions:

1. What is the relative importance of individual-related characteristics and of general earnings instability as factors behind overall earnings inequality? In other words, even though individual characteristics are important determinants of inequality (Menezes Filho, 2001), the proposed methodology allows estimating the relative contribution of these factors vis-à-vis the magnitude of inequality resulting from earnings instability.

2. With regard to inequality resulting from individual characteristics, what is the relative importance of factors related to observed and unobserved heterogeneity? Given that several studies consider education to be a key determinant of inequality (ibid), the approach used in this paper allows comparing observed heterogeneity aspects (e.g., education and age) with unobserved ones (e.g. ability). This is done by comparing the estimate of the earnings individual component with its estimate obtained from earnings residuals netted out of education and age effects.

3. Finally, one may check whether an increase in inequality over a given time interval is due mostly to an increase in the individual component or, alternatively, to an increase in the transitory component of inequality. Moreover, even if the earnings inequality had remained roughly constant, the relative importance of its determinants still might have varied. This is of note since the individual component of inequality can be regarded as permanent, long-run inequality. Therefore, the 
larger the permanent component (and consequently the lesser the instability), the smaller earnings mobility will be. In the specific context of this paper, mobility should be understood as position shift between individuals across the earnings distribution.

Furthermore, identifying the factors that underlie inequality has important implications with regard to public policy: a greater magnitude of the permanent component suggests the importance of policies targeted, for instance, at workforce qualification, whereas a large transitory component suggests the importance of policies that somehow try to offset the shocks experienced by individuals in the labor market.

This paper is structured as follows. Section 2 introduces the variance component models and describes their properties. Section 3 deals with the database and sample selection. Section 4 shows the empirical results. Section 5 presents the final remarks.

\section{Variance Component Models}

The aim of variance component models is to decompose the autocovariance structure of earnings into its transitory and permanent components. This type of exercise allows determining to what extent earnings inequality over time results from permanent individual characteristics or from idiosyncratic shocks.

The permanent component of earnings is typically associated with productivity or human capital, whereas the transitory component is normally related to noise derived from economic instability. Thus, the permanent component is associated with the earnings profile of an individual's life cycle, whereas the transitory component represents stochastic fluctuations about this profile. Actually, the greater the magnitude of the permanent component, the smaller the mobility and, therefore, the larger the long-run earnings inequality.

In other words, an increase in inequality can be due to (i) an increase in the earnings of the individual component, in which the ordering in the earnings distribution is maintained while dispersion increases; or to (ii) a general increase in earnings instability, in which individuals swap positions across the earnings distribution, i.e., mobility exists. ${ }^{1}$

The simplest variance component model, dubbed as canonical model, can be formulated as follows (Ramos, 2003):

$$
y_{i t}=\mu_{i}+v_{i t}
$$

\footnotetext{
${ }^{1}$ According to Ramos (2003), permanent differences imply little or no earnings mobility and a possibly rigid labor market in which the initial position is essential for determining earnings profiles of an individual's life cycle and his/her position in earnings distribution across time. The transitory component, on the other hand, indicates earnings instability, or "year to year 'churning' through the ranks of the annual earnings distribution", according to Baker and Solon (1999, p. 2). Therefore, the transitory component indicates mobility.
} 
where $y_{i t}$ is some measure of individual $i$ 's earnings at time $t$, modeled as the sum of a time-invariant individual component which is uncorrelated among individuals, $\mu_{i} \sim i i d\left(\bar{\mu}_{i}, \sigma_{\mu}^{2}\right)$, plus a pure white-noise transitory component, $v_{i t} \sim i i d\left(0, \sigma_{v}^{2}\right)$.

Under the additional hypothesis of orthogonality between the permanent and transitory components, which leads to $\operatorname{Cov}\left(\mu_{i}, v_{i t}\right)=0$, the following theoretical covariance structure can be derived:

$$
\begin{aligned}
\operatorname{Var}\left(y_{i t}\right) & =\sigma_{\mu}^{2}+\sigma_{v}^{2} \\
\operatorname{Cov}\left(y_{i t}, y_{i s}\right) & =\sigma_{\mu}^{2}, t \neq s
\end{aligned}
$$

As can be seen, the simplicity of the canonical model results in a covariance structure with a very straightforward interpretation: the overall variance of the logarithm of earnings is equivalent to the sum of the variances of the permanent and transitory components. With regard to covariances, as the transitory component is a white noise, all that is left as covariance between any two periods is the variance of the permanent component only - which does not vary over time or in terms of lag order between two periods.

As the reader may observe, the covariance structure in (2) is similar to the random effects structure described by Wooldridge (2002). In fact, as shall be hereafter explained, this similarity is merely formal, for both models reside on quite different sets of hypothesis. As it goes, Wooldridge's discussion about fixed versus random effects in the context of error component models aims to provide consistent estimates for the coefficients of a panel regression in the presence of omitted variables. For that reason, hypotheses about the correlation between the unobserved heterogeneity component and the remaining explanatory variables are necessary, in addition to the hypothesis of strict exogeneity of explanatory variables relative to the idiosyncratic shock. ${ }^{2}$ Those hypotheses are not necessary in this study because the analysis is not based on observed explanatory variables: rather, the variance decomposition is applied directly to the explained variable (earnings). Later on, the decomposition is applied to the residual of a regression on education and age dummies; but, even in this case, the hypotheses raised by Wooldridge are not necessary, since the aim is not to assess the $\boldsymbol{\beta}$ of a regression.

Finally, the simplicity of the canonical model imposes quite rigid restrictions on the dynamic structure of earnings. Thus, the literature on variance components has sought to provide more comprehensive specifications for the autocovariance structure of earnings. In this regard, the proposed models depart progressively

\footnotetext{
${ }^{2}$ Note that Wooldridge refers to a specific model, given the hypotheses above, as fixed effect model. Differently from the common use, Wooldridge does not refer to term $\mu_{i}$ as "fixed effect" but as "unobserved heterogeneity" instead, since this term can be used in the fixed effect model or in the random effect model, constituting a random variable in both cases. Therefore, the terminology used in this paper follows Wooldridge's.
} 
from the simple structure of the canonical model, in an attempt to capture additional relevant aspects of the earnings dynamics.

Basically, the extensions developed in the literature postulate some type of stochastic process for both the permanent and transitory components based on reasonable hypotheses about the dynamic structure of earnings, in addition to postulating also some kind of initial conditions for models with recursive structures.

MaCurdy (1982) introduces a more general specification that summarizes the specifications described in previous studies. His model combines a factor model for the permanent component and a time series model for the transitory component:

$$
\begin{aligned}
y_{i t} & =\lambda_{t}^{\prime} f_{i}+v_{i t} \\
\lambda_{t}^{\prime} & =\left(\lambda_{1 t}, \ldots, \lambda_{g t}\right) ; f_{i}^{\prime}=\left(f_{1 i}, \ldots, f_{g i}\right) \\
a(L) v_{i t} & =m(L) \xi_{i t}
\end{aligned}
$$

where $a(L)$ and $m(L)$ are polynomials of the lag operator $L$ and $\xi_{i t}$ is white noise. Thus we have a model of $g$ factors combined with an $\operatorname{ARMA}(p, q)$ model. As an example, if we take $\lambda_{1 t}=1$ and $\lambda_{2 t}=t$ and the remaining $\lambda_{i t}=0, i=3, \ldots, g$; we will have the random growth model for the permanent component with an ARMA $(p, q)$ process for the transitory component. Note that this specification also allows for time-varying parameters for the permanent component (known as factor loadings or factor weights).

In their seminal study, Moffit and Gottschalk (1995) add, relatively to MaCurdy, the possibility of a random walk specification for the permanent component, and include time-varying parameters for the ARMA process. Observe that the random walk leads to a linear growth pattern in the life cycle for variances, whereas the random growth model results in a quadratic pattern. The authors estimated the following specification:

$$
\begin{aligned}
y_{i a t} & =\alpha_{t} \mu_{i a t}+v_{i a t} \\
\mu_{i a t} & =\mu_{i, a-1, t-1}+\omega_{i a t} \\
v_{i a t} & =\rho_{t} v_{i, a-1, t-1}+\xi_{i a t}+\theta_{t} \xi_{i, a-1, t-1}
\end{aligned}
$$

Index "a" refers to ages or cohorts. In this model earnings vary both over the individual life cycle and over calendar time. Parameter $\alpha_{t}$ can be interpreted as the (time-varying) price of latent unobserved human capital. This decomposition defines permanent shocks as those that are non mean-reverting (due to the random walk, a nonstationary process) and transitory shocks as those that are meanreverting (due to ARMA, a stationary process).

In brief, modeling possibilities found in the literature vary along the following lines: 
1. For the permanent component, there are basically two possibilities to generalize the simple individual effect: firstly, by means of a random growth model formulation, which results in a permanent income profile with individual intercepts and slopes (which may contain a quadratic term to allow for a curvature in the profile). Alternatively, through a random walk model, which also generates a nonstationary pattern for permanent variances, but linear rather than quadratic (as in the random growth model). In addition, it is possible to add time-varying parameters which load the permanent component as a whole.

2. With regard to the transitory component, the white noise of the canonical model is usually replaced with specifications that allow for serial correlation in this component, so as to captures the persistence of shocks, which is obtained by an ARMA process. It is also possible to add factor loadings on the transitory component as a whole, or time-varying parameters for the ARMA process.

3. With respect to initial conditions, there are basically two alternatives: either one reduces the variance of the initial transitory component to the variance of its shock (white noise) - as Lillard and Willis (1978) or Moffit and Gottschalk (1995) did; or one separately estimates an initial variance for the transitory component in order to capture the accumulation of the stochastic process up to the year preceding the beginning of the panel data analysis - as in Baker (1997), Baker and Solon (1999), or Cappellari (2000).

As to the results found in the literature, one should initially note that most studies carried out using the proposed method included OCDE countries, in which inequality showed a pronounced increase in the 1970s. In a U.S. study, Moffit and Gottschalk (1995) observed that half of the increase in variance between 1969 and 1987 can be attributed to each component. Dickens (2000) found similar results for England between 1975 and 1995. Baker and Solon (1999) also observed an increase in both components for Canada, but they found a larger increase in the permanent component.

In Italy, Cappellari (2000) used earnings residuals and concluded that the permanent component shows a heavier weight (ranging from $2 / 3$ to virtually $100 \%$ of the overall variance), but, on the other hand, the transitory component, and therefore the earnings instability, increases over the period. Gustavsson (2002) concluded that the increase in inequality observed in Sweden between 1991 and 1999 is due to the permanent component (for older cohorts) or to the transitory component (for younger cohorts). Biewen (2002) carried out a study in which he analyzed data for Germany in the 1980s and 1990s based on models for the residual and found out that $52 \%$ to $69 \%$ of inequality in Western Germany was due to individual factors, whereas in Eastern Germany this component grew from $20 \%$ in 
1990 to over $70 \%$ in 1998. Finally, Ramos (2003) observed that in Great Britain (1991-1999), there was an increase in the transitory component over the decade, which actually outperformed the permanent component in terms of relative importance. The use of residuals strongly affects the decomposition: the transitory component prevails throughout the period, and the relative contribution of each factor changes relatively little.

The only study concerning developing countries we found is the one Freije and Souza (2001) conducted for Venezuela (1995-1997). The authors observed an $80 \%$ increase in the transitory component (the difference between earnings and residuals is quite small), which indicates the importance of earnings instability as a component of inequality. Nevertheless, the result strongly contradicts those obtained for industrialized countries, in which the permanent component often plays a more important role. As we will discuss later in further detail, our results are closer to those obtained for industrialized countries than those for Venezuela. In this regard, one should recall that our analysis deals only with the formal sector of the state of São Paulo, whose characteristics are very peculiar when compared to the rest of Brazil (e.g.: average income greater than and Gini coefficient smaller than those of Brazil as a whole, as reported by Hoffmann (2002)).

As to other Brazilian studies on inequality, authors such as Barros et al. (2001) and Hoffmann (2002) show that inequality varied slightly in Brazil in the 1990s, considering all types of earnings (not only from labor). Menezes Filho (2001) found education to be the main determinant of inequality. Our study, as will be shown further ahead, confirms that inequality remains stable and that individual aspects prevail; however, unobserved heterogeneity plays a key role in the individual component of inequality.

\section{Database and Sample Selection}

This study uses RAIS official data, based on a questionnaire issued by the Brazilian Ministry of Labor and Employment, which must be completed by each and every formal establishment in the country. Thus, RAIS represents only the formal sector in the Brazilian labor market.

The state of São Paulo, including both the state capital and the countryside, was used as geographic base. A problem with this base is that one cannot keep track of individuals who migrate from the state. This means that our analysis is conditional on individuals who hold a job in the formal sector of the state of São Paulo throughout the period under analysis. Additionally, in line with the literature, we kept in the sample only men of working age, i.e., those who had finished their education and were not about to retire. These inclusion criteria may, indeed, result in peculiarities with regard to what could be observed in Brazil as a whole; therefore, the problem of sample selection is of great relevance for the interpretation of our results, as we shall presently explain.

According to Wooldridge (2002), a non-random sample may arise (i) from the 
study design itself, or (ii) from the behavior of sampled individuals. The exclusion of women, students, and retirees from the sample is a way to overcome the bias associated with the second aspect, i.e., the differentiated decision of these groups to participate in the labor market.

Nonetheless, as observed by Wooldridge, the sample selection can only be a problem if the target population has been carefully specified. "If we are interested in a subset of a larger population, then the proper approach is to specify a model for that part of the population, obtain a random sample from that part of the population, and proceed with standard econometric methods" (ibid, p. 551).

Based on these observations, the results of this study are valid inferences for the population of prime-age male workers who worked in the formal sector of the state of São Paulo throughout the study period. Inferences about the Brazilian labor market as a whole should be made with extreme caution and are not recommended at all.

In this study, we used data for the 1990-1998 period - i.e, for almost the entire decade, except for 1999. Based on the exclusion criteria mentioned above, we obtained 3.2 to 3.7 million observations for each year, which resulted in a balanced panel with 342,450 individual observations per year/cross section. Some characteristics of our final sample are shown in the Appendix: log of average real earnings, maximum and minimum earnings for each year, and also the distribution of educational characteristics and ages for each year.

As to the variable under study, we used the Neperian logarithm of real earnings for every December (in Brazilian currency value as of June 2004, deflated by the Brazilian consumer price index, calculated by the Brazilian Institute of Geography and Statistics: INPC/IBGE). ${ }^{3}$ This variable corresponds to the total labor earnings not adjusted by the number of hours worked. Our final study variable was, however, the individual deviation from the annual average. Analogously to Baker and Solon (1999):

$$
\begin{aligned}
Y_{i t} & =\alpha_{t}+y_{i t} \\
y_{i t} & =\mu_{i}+v_{i t}
\end{aligned}
$$

where $\mathrm{Y}_{i t}$ is the natural logarithm of the real earnings of individual $i$ at time $t, \alpha_{t}$ is the mean between individuals at $t$ of the $l n$ of the real earnings, and $y_{i t}$ represents the individual deviation from that average, that is, a measure of

\footnotetext{
${ }^{3}$ From our database, we could use both the earnings paid in December and the average annual earnings. The decision to use the earnings paid in December to the detriment of the average earnings is due to the fact that the averaging of earnings would eliminate the transitory component, which would seriously affect the decomposition. In fact, both variables overestimate overall inequality: the average earnings include observations of zero earnings, while the earnings paid in December embed a "square" effect of variance on a linear pay rise. Anyway, the increase in variance in the latter case is much more systematic and predictable and has the major advantage of presumably not affecting the decomposition.
} 
relative earnings. The latter is the variable used in our empirical study, which we will hereinafter refer to as simply "earnings". This variable also has the advantage of simplifying the calculations of variances and covariances, since its mean is zero by construction.

In addition to the earnings variable, we are going to analyze the residuals of an earnings regression. Given that a residual represents the earnings controlled by observed heterogeneity, it is interesting to compare the decomposition of earnings variances with the decomposition of residuals' variances. Presumably, the permanent component should have a smaller effect on the decomposition of residuals, and the difference can be interpreted as the contribution of observed heterogeneity to inequality.

\section{Estimated Models and Results}

\subsection{Decomposition of earnings variances}

\subsubsection{Empirical variance-autocovariance matrix}

Considering the balanced panel dataset for all ages between 25 and 64 years, it is possible to obtain the following empirical variance-covariance matrix for relative earnings:

Table 1

Earnings variance-covariance matrix

\begin{tabular}{l|ccccccccc}
\hline & 1990 & 1991 & 1992 & 1993 & 1994 & 1995 & 1996 & 1997 & 1998 \\
\hline 1990 & 0.62 & & & & & & & & \\
1991 & 0.55 & 0.67 & & & & & & & \\
1992 & 0.54 & 0.6 & 0.69 & & & & & & \\
1993 & 0.53 & 0.59 & 0.62 & 0.69 & & & & & \\
1994 & 0.52 & 0.57 & 0.59 & 0.61 & 0.68 & & & & \\
1995 & 0.5 & 0.54 & 0.56 & 0.57 & 0.59 & 0.64 & & & \\
1996 & 0.5 & 0.53 & 0.56 & 0.57 & 0.58 & 0.58 & 0.66 & & \\
1997 & 0.5 & 0.54 & 0.56 & 0.58 & 0.57 & 0.57 & 0.61 & 0.68 & \\
1998 & 0.49 & 0.52 & 0.54 & 0.55 & 0.55 & 0.56 & 0.58 & 0.6 & 0.66 \\
\hline \multicolumn{7}{l}{ Source: elaborated by the authors. }
\end{tabular}

Variance, our measure of inequality, oscillated throughout the period without considerably changing its pattern - a quite different pattern from the sustained increase observed in countries such as England and the United States (Ramos (2003); Moffit and Gottschalk (1995)).

The autocovariances, which indicate a pattern of dynamic, linear earnings dependence, show a pattern that is often found in the literature: they decrease quickly during the first lags and decrease more slowly in larger lags. This pattern may suggest an ARMA scheme for the transitory component (MA for explaining the quick initial reduction, as MA is a memory-free process, and AR for explaining the persistence of shocks on the long tail), plus a time-invariant individual component (for explaining the asymptote, since there exists only the individual 
component between sufficiently distant periods, and the effect of AR is expected to disappear).

Figure 1 shows the changing pattern of variance, which is also observed for the short-term linear dependence of earnings (covariances with lag equal to 1). The covariances with a lag equal to 4 do not show a clear time pattern:

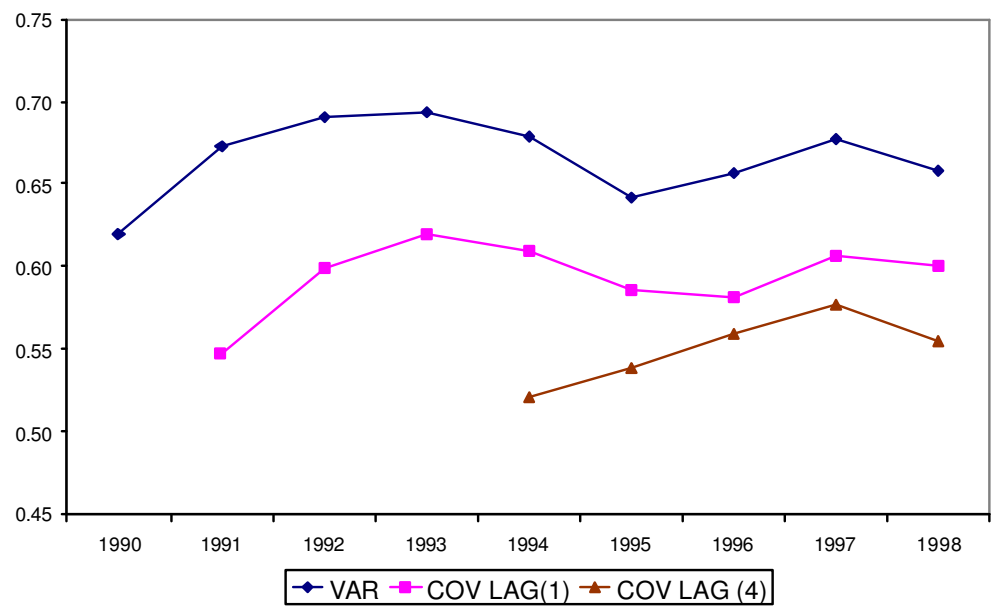

Source: elaborated by the authors

Figure 1

Empirical variances and autocovariances of earnings

Thus, given this structure of the observed autocovariances, our aim is to estimate a variance component model that appropriately fits this structure. The estimates are shown in what follows. 


\subsubsection{Estimates for variance component models}

As a preliminary exercise, we estimated the canonical model. For convenience, its structure is reproduced below:

$$
\begin{aligned}
\operatorname{Var}\left(y_{i t}\right) & =\sigma_{\mu}^{2}+\sigma_{v}^{2} \\
\operatorname{Cov}\left(y_{i t}, y_{i s}\right) & =\sigma_{\mu}^{2}
\end{aligned}
$$

The equally weighted minimum distance (EWMD) method was used for the estimation (see Appendix). The results for the canonical model are shown below in the first column of Table $2:^{4}$

Table 2

Estimates for models of earnings variances

\begin{tabular}{l|cc}
\hline & canonical & random growth \\
\hline $\operatorname{Var}(\mu)$ & $0.5584(0.0012)$ & $0.4833(0.0000)$ \\
$\operatorname{Var}(\beta)$ & & $0.0011(0.0002)$ \\
$\operatorname{Cov}(\mu, \beta)$ & & $-0.0042(0.0000)$ \\
$\operatorname{Var}(v)$ & $0.1071(0.0003)$ & \\
$\operatorname{Var}(\xi)$ & & $0.0945(0.0000)$ \\
$\operatorname{Var}\left(v_{-1}\right)$ & $0.7827(0.0001)$ \\
$\rho$ & $0.2299(0.0001)$ \\
$\lambda_{1}$ & $1.0882(0.0001)$ \\
$\lambda_{2}$ & $1.1242(0.0000)$ \\
$\lambda_{3}$ & $1.1318(0.0002)$ \\
$\lambda_{4}$ & & $1.1162(0.00003)$ \\
$\lambda_{5}$ & & $1.0791(0.0002)$ \\
$\lambda_{6}$ & & $1.0869(0.00003)$ \\
$\lambda_{7}$ & & $1.959(0.00015)$ \\
$\lambda_{8}$ & $0.0662(0.00002)$ \\
obj.f. & 0.0434 & 0.0007 \\
chi-stat & 76877.0 & 7453.1 \\
\multicolumn{2}{l}{ Standard deviations in brackets. } \\
\multicolumn{2}{l}{ Source: elaborated by the authors. }
\end{tabular}

Both parameters are statistically significant. Figure 2 shows that the variance estimated using the canonical model crosses the path of observed variances about its mean:

\footnotetext{
${ }^{4}$ The estimates were computed with OxGauss.
} 


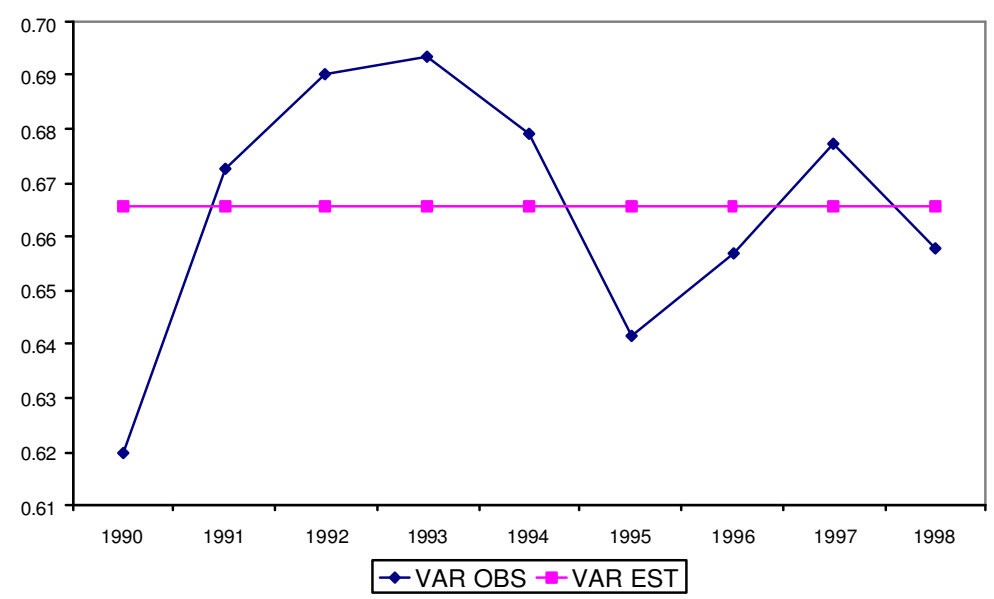

Source: elaborated by the authors

Figure 2

Estimated variance - canonical model

The variance decomposition obtained from the model indicates a much larger participation for the permanent variance: $84 \%$ of the predicted variance versus $16 \%$ for the transitory component. This indicates that permanent inequality accounts for the largest share of overall inequality and that the inequality resulting from pure shocks is relatively unimportant. However, its chi-square statistics indicates rejection of the model's structure, consequently requiring the estimation of more general models.

After experimenting with several different specifications, our preferred one turned out to be a random growth model with factor loadings for the permanent component and an $\mathrm{AR}(1)$ process for the transitory component. ${ }^{5}$ The initial condition of the AR process is shown on the additional parameter $\operatorname{Var}\left(v_{i-1}\right)=\sigma_{-1}^{2}$, which indicates the accumulation of the stochastic process up to the year before the sampling period. Its equation is as follows:

$$
\begin{aligned}
& y_{i t}=\lambda_{t}\left[\mu_{i}+\beta_{i} t\right]+v_{i t} \\
& v_{i t}=\rho v_{i t-1}+\xi_{i t}
\end{aligned}
$$

The implied covariance structure is the following: ${ }^{6}$

${ }^{5}$ Several specifications were estimated using the random walk or random growth with ARMA, with or without time-varying parameters. The choice of our preferred model is based on the lowest chi-square statistics, in line with the usual procedures described in the literature.

${ }^{6}$ The derivation of the covariance structures can be requested from the authors. 


$$
\begin{aligned}
\operatorname{Var}\left(y_{i t}\right) & =\lambda_{t}^{2}\left(\sigma_{\mu}^{2}+t^{2} \sigma_{\beta}^{2}+2 t \sigma_{\mu \beta}\right)+\rho^{2(t+1)} \sigma_{-1}^{2}+\sum_{i=0}^{t} \rho^{2 i} \sigma_{\xi}^{2} \\
\operatorname{Cov}\left(y_{i t}, y_{i t+s}\right) & =\lambda_{t} \lambda_{t+s}\left(\sigma_{\mu}^{2}+t \sigma_{\mu \beta}+(t+s) \sigma_{\mu \beta}+t(t+s) \sigma_{\beta}^{2}\right) \\
& +\rho^{s}\left(\rho^{2(t+1)} \sigma_{-1}^{2}+\sum_{i=0}^{t} \rho^{2 i} \sigma_{\xi}^{2}\right)
\end{aligned}
$$

The result of estimates for this model can be seen in the second column of Table 2. All parameters are significant and the chi-square statistics has the smallest value among all estimated models.

The covariance between individual intercepts and slopes is negative, which indicates a trade-off between initial permanent earnings and their later growth rate - which is actually counterintuitive given what one should expect in the case of Brazil. Nevertheless, its absolute value, albeit statistically significant, is quite low. Also note that the factor loadings (latent price or permanent component premium) show a remarkable increase in comparison to their initial value (normalized to 1 in 1990). Even with a relative reduction to its peak in 1998, its value at the end of the period is still higher than the initial value of 1 .

Figures 3, 4 and 5 show the adjustment for this model, closely mimicking the observed variances and covariances:

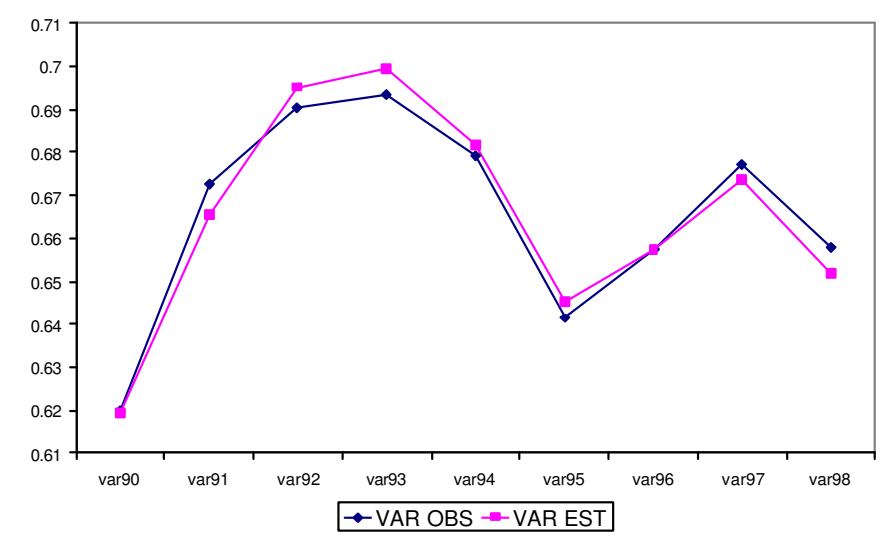

Source: elaborated by the authors

Figure 3

Estimated variance - random growth with factor loadings $+\mathrm{AR}(1)$ 


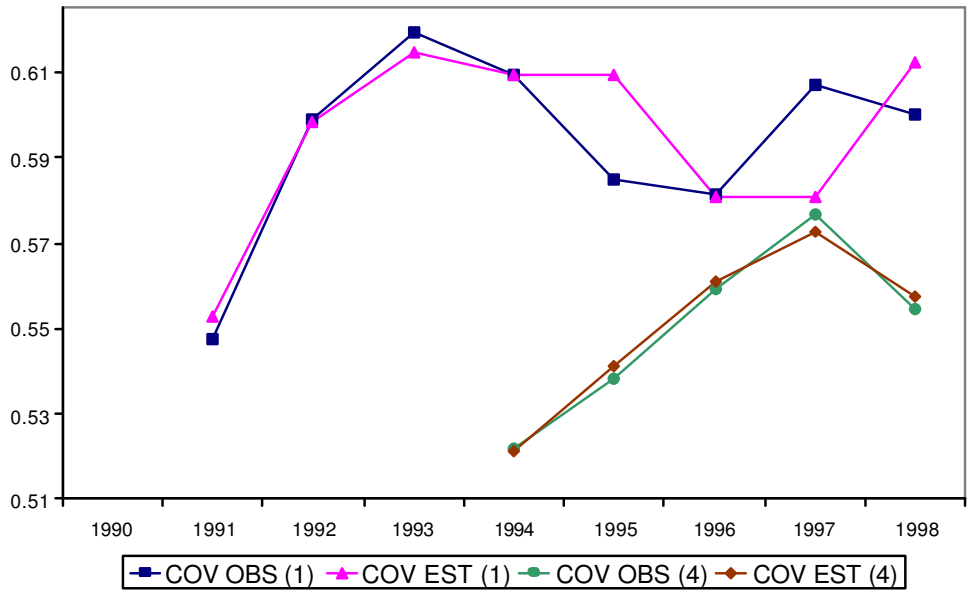

Source: elaborated by the authors

Figure 4

Estimated covariance - random growth with factor loadings $+\mathrm{AR}(1)$

Our preferred model results in the following variance decomposition:

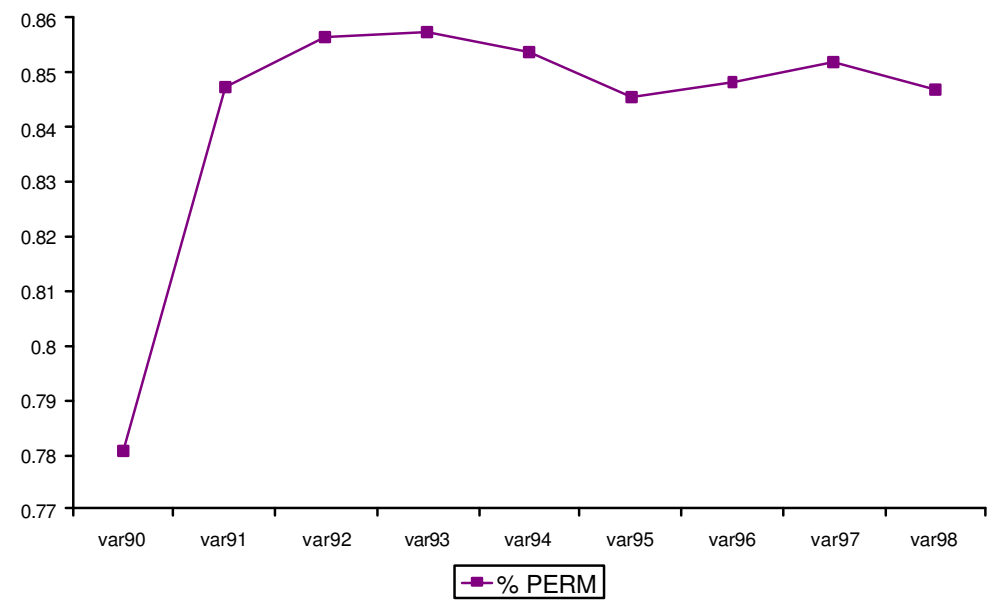

Source: elaborated by the authors

Figure 5

Variance decomposition - random growth with factor loadings + AR(1) 
We show only the percentage of the estimated variance due to the permanent component. The initial leap occurs by the construction of the model: at $t=0$ the term $\beta_{i}^{*} t$ (eq. 06) disappears, which leads to a greater participation of transitory variance at the initial time. The weight of the permanent variance oscillates smoothly throughout the period, always around $85 \%$ of the total variance, which implies an equally oscillating pattern for the weight of the transitory variance, around $15 \%$ of the total. This magnitude for the permanent variance is indeed quite similar to the one observed in the canonical model.

\subsubsection{Residual models}

The aim of this section is to estimate variance component models for earnings residuals so as to assess how different variance decomposition would be relative to the decomposition previously observed in earnings models. The residuals are obtained from ordinary least squares regressions, for each cross-section separately. The dependent variable is the ln of real earnings, which is regressed against age and education dummies. It is not within our scope to analyze regression coefficients; our aim is merely to obtain the earnings net of age and education effects, which is precisely the residual of the earnings regression. After that, we are going to apply the same variance decomposition methods used for the earnings directly to the earnings residual.

The empirical variance-covariance matrix for the earnings residuals is shown below:

Table 3

Residuals' variance-covariance matrix

\begin{tabular}{l|ccccccccc}
\hline & 1990 & 1991 & 1992 & 1993 & 1994 & 1995 & 1996 & 1997 & 1998 \\
\hline 1990 & 0.44 & & & & & & & & \\
1991 & 0.36 & 0.49 & & & & & & & \\
1992 & 0.34 & 0.4 & 0.49 & & & & & & \\
1993 & 0.33 & 0.38 & 0.41 & 0.49 & & & & & \\
1994 & 0.31 & 0.35 & 0.37 & 0.4 & 0.47 & & & & \\
1995 & 0.29 & 0.32 & 0.44 & 0.35 & 0.37 & 0.44 & & & \\
1996 & 0.29 & 0.32 & 0.34 & 0.36 & 0.36 & 0.37 & 0.47 & & \\
1997 & 0.28 & 0.31 & 0.33 & 0.35 & 0.35 & 0.35 & 0.39 & 0.47 & \\
1998 & 0.26 & 0.29 & 0.31 & 0.32 & 0.32 & 0.33 & 0.36 & 0.38 & 0.45 \\
\hline \multicolumn{7}{l}{ Source: elaborated by the authors. }
\end{tabular}

As expected, the variance and covariance levels in all cells are lower than those observed for the earnings variable in Table 1 (they represent around 60 to $70 \%$ of these values). After deducting the effects of education and age, the levels of inequality (variances) and the persistence of earnings (autocovariances) are lower.

The patterns observed in the matrix are similar to those in Table 2, both for variances (which are oscillating) and covariances (which decrease as the lag increases and oscillate in the time series of covariances for fixed lags).

In order to compare the effects of variance decomposition on earnings and 
residuals, we re-estimated both the canonical model and the random growth model with factor loadings. Our preferred model in the case of residuals, however, is slightly different, since it includes an $\operatorname{ARMA}(1,1)$ specification instead of $\operatorname{AR}(1)$ for the transitory component. The permanent component continues to be represented by a random growth model plus factor loadings. ${ }^{7}$ Table 4 shows the estimates of variance component models for residual variance:

Table 4

Estimates of models for (residual) Var

\begin{tabular}{l|cc}
\hline & canonical & random growth \\
\hline $\operatorname{Var}(\mu)$ & $0.3415(0.0009)$ & $0.2404(0.0003)$ \\
$\operatorname{Var}(\beta)$ & & $0.0009(0.0000)$ \\
$\operatorname{Cov}(\mu, \beta)$ & & $-0.0026(0.0000)$ \\
$\operatorname{Var}(v)$ & $0.1263(0.0003)$ & \\
$\operatorname{Var}(\xi)$ & & $0.0010(0.0000)$ \\
$\operatorname{Var}\left(v_{-1}\right)$ & & $0.6233(0.0008)$ \\
$\rho$ & $0.4039(0.0027)$ \\
$\theta$ & & $-10.3997(0.000)$ \\
$\lambda_{1}$ & $1.2127(0.0013)$ \\
$\lambda_{2}$ & $1.2527(0.0008)$ \\
$\lambda_{3}$ & $1.2666(0.0011)$ \\
$\lambda_{4}$ & & $1.2164(0.0001)$ \\
$\lambda_{5}$ & & $1.1493(0.0014)$ \\
$\lambda_{6}$ & & $1.1758(0.0011)$ \\
$\lambda_{7}$ & & $1.1652(0.0021)$ \\
$\lambda_{8}$ & 0.0486 & $1.0948(0.0018)$ \\
obj.f. & 90975 & 0.0012 \\
chi-stat & & 8272.8 \\
\hline \multicolumn{2}{l}{ Standard deviations in brackets. } \\
Source: elaborated by the authors.
\end{tabular}

What does variance decomposition look like in these models? For the canonical model, the weight of the permanent component drops from $84 \%$ (earnings variable) to $73 \%$ (residual variable). This decrease was actually expected: after discounting the effects of education and age, the weight of the permanent component should fall. However, even if the observed decrease is not negligible, the magnitude of the permanent component is still quite high. This means that a larger share of inequality may be attributed to individual factors not included in our regression - possibly factors of unobserved heterogeneity, such as ability. Anyway, one should always bear in mind that our data refer to the formal labor market (CLT), and therefore has institutional characteristics which influence participation in this market.

Our preferred specification for residuals is a slightly extended version in relation to the specification preferred for earnings, consisting of a random growth model

\footnotetext{
${ }^{7}$ The AR(1) specification had to be cast aside, since the program provided negative estimates to some variances, which does not make sense. The inclusion of the MA term does not change substantially the comparability of results as the aim is to match the decomposition of the best model for earnings and for residuals, and the models chosen for each variable are quite similar, anyway.
} 
plus factor loadings for the permanent component, and an $\operatorname{ARMA}(1,1)$ process for the transitory component. Its equation is as follows:

$$
\begin{aligned}
& y_{i t}=\lambda_{t}\left[\mu_{i}+\beta_{i} t\right]+v_{i t} \\
& v_{i t}=\rho v_{i t-1}+\xi_{i t}+\theta \xi_{i t-1}
\end{aligned}
$$

One can derive the following covariance structure from it:

$$
\begin{aligned}
\operatorname{Var}\left(y_{i t}\right) & =\lambda_{t}^{2}\left(\sigma_{\mu}^{2}+t^{2}+\sigma_{\beta}^{2}+2 t \sigma_{\mu \beta}\right)+\gamma_{t}^{2} \operatorname{Var}\left(v_{i t}\right) \\
\operatorname{Cov}\left(y_{i t}, y i t+s\right) & =\lambda_{t} \lambda_{t+s}\left(\sigma_{\mu}^{2}+t \sigma_{\mu \beta}+(t+s) \sigma_{\mu \beta}+t(t+s) \sigma_{\beta}^{2}\right) \\
& +\gamma_{t} \gamma_{t+s}\left(\rho^{s} \operatorname{Var}\left(v_{i t}\right)+\rho^{s-1} \theta \sigma_{\xi}^{2}\right)
\end{aligned}
$$

where $\operatorname{Var}\left(v_{i t}\right)$ is given by:

$$
\operatorname{Var}\left(v_{i t}\right)=\left\{\rho^{2(t+1)} \sigma_{-1}^{2}+\sigma_{\xi}^{2}\left[\left(\sum_{i=0}^{t} \rho^{2 i}\right)\left(1+\theta^{2}\right)+2 \rho^{1+2 t} \theta\right]\right\}
$$

The estimates for this model are shown in the second column of Table 4. All of the variables are significant, and the chi-square statistics has the lowest value among all estimated models. Again, note that the covariance between the intercept and the slope of the random growth is estimated with a negative value and that the factor loadings show a much more remarkable growth. The adjustment of the model is shown in Figure 6:

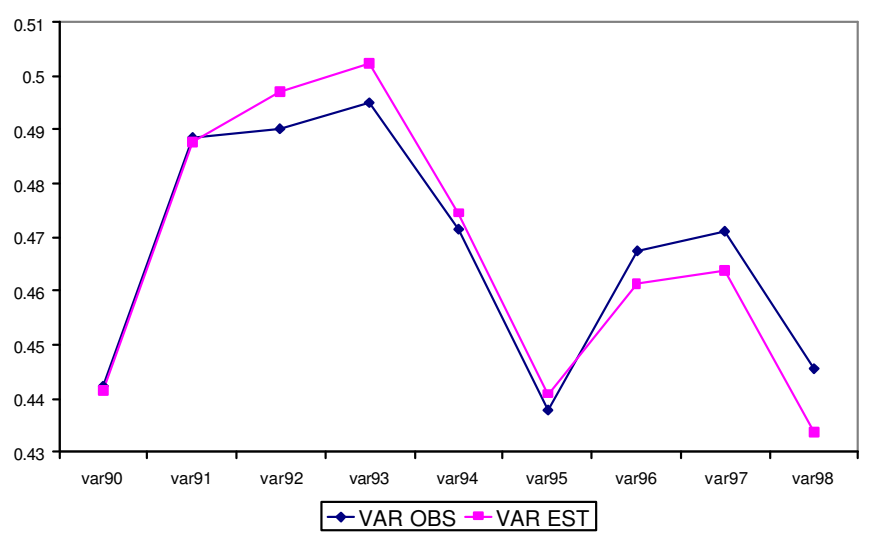

Source: elaborated by the authors

Figure 6

Estimated variances - random growth with factor loadings $+\operatorname{ARMA}(1,1)$ 


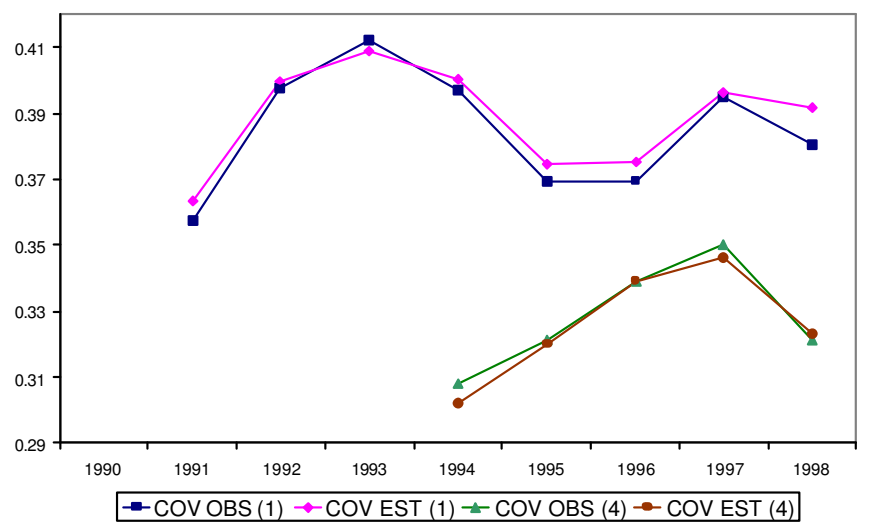

Source: elaborated by the authors

Figure 7

Estimated covariances - random growth with factor loadings $+\operatorname{ARMA}(1,1)$

The variance decomposition is shown in Figure 8:

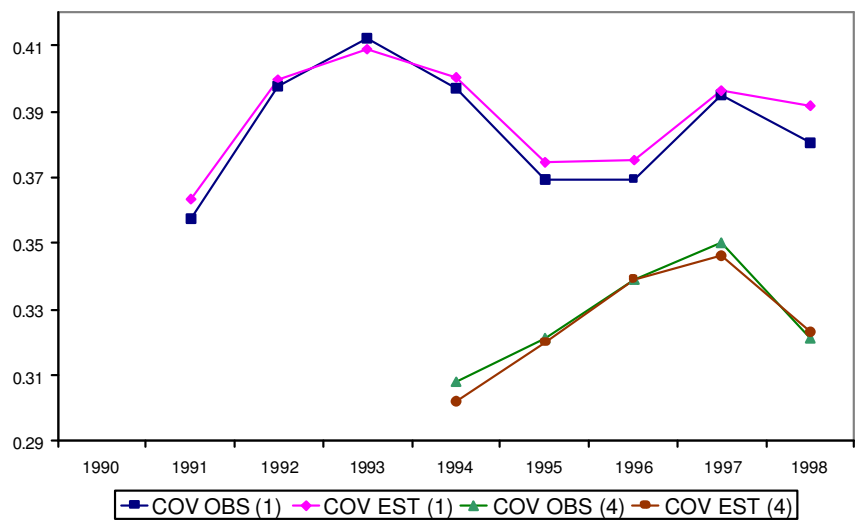

Source: elaborated by the authors

Figure 8

Variance decomposition - Random growth with factor loadings + ARMA(1,1) 
As before, the permanent component plays a major role in total variance. Just as in the canonical model, there is a sharp decrease in the weight of the permanent component - around 10 to 15 percentage points - compared to that which was observed for earnings.

In brief, the change in decomposition related to the use of earnings or residuals as dependent variable can be illustrated as follows: in the canonical model, the permanent component of earnings variance was 0.5584 . Now, the permanent component of residual variance is 0.3415 . The share of the permanent component associated with education and age is equal to $1-(0.3415 / 0.5584)$, which corresponds to nearly $39 \%$. Similar calculations using the preferred model indicate that education and age are associated with $38 \%$ in 1991 and $43 \%$ in 1998 of permanent heterogeneity among individuals.

\section{Conclusion}

The stability and high level of inequality in Brazil, as well as the key role of education, are widely documented in the Brazilian literature (Barros et al. (2001); Souza (2002); Hoffmann (2002), Menezes Filho (2001)). As a matter of fact, there has not been a remarkable and recent increase in inequality in Brazil whose causes would require explanation, as in OCDE countries which produced the literature on variance components. Due to such aspects, what are the contributions of this paper?

First, it was possible to find out that there is a permanent component with large magnitude and great stability underneath the great stability of inequality. This means not only that the position of individuals in the ranking of income distribution depends mainly on their personal characteristics, but also that the initial position of an individual in this distribution determines his/her position throughout his/her career, since there exists very little mobility (swap of positions) among individuals across the earnings distribution. In other words, individual characteristics are accountable for better pay and also for a permanently better pay, since earnings instability is small.

Secondly, our study revealed that most of the permanent component is due to unobserved heterogeneity factors. In fact, this result runs counter to the general assumption that education is the major determinant of inequality.

Notwithstanding, our results should be qualified in terms of the special characteristics of the sample used. Prime-age male workers who hold a job in the formal sector of the state of São Paulo certainly represent the lower bound of the transitory component in Brazil. The sample includes the possibly most stable earnings in the whole private sector. In this regard, one may take for granted that the preponderance of the individual component is associated with the system's institutional characteristics; in particular, hiring in the formal market of the state of São Paulo works as a large filter individuals with presumably initial good educational level sift through. Moreover, the low level of earnings instability should also 
reveal characteristics that are peculiar to the Brazilian labor regulations, represented by the Consolidation of Labor Laws (CLT). In this regard, it is no surprise that our results resemble those obtained by industrialized countries rather than those obtained by Freije and Souza (2001) for Venezuela, until then the only study carried out for developing countries, which showed the importance of the earnings instability factor.

In terms of public policy, the conclusions of this study suggest workforce qualification policies so as to minimize the earnings dispersion produced by the premium paid to human capital, as well as the search for formal jobs generating mechanisms, so as to provide the population in general with the benefits of the lower earnings instability observed in the sector.

\section{References}

Abowd, J. \& Card, D. (1989). On the covariance structure of earnings and hours changes. Econometrica, 57.

Altonji, J. \& Segal, L. (1996). Small sample bias in GMM estimation of covariance structures. Journal of Business \& Economic Statistics, 14(3).

Atkinson, A. B., Bourguignon, F., \& Morrison, C. (1992). Empirical Studies of Earnings Mobility. Harwood Publishers.

Baker, M. (1997). Growth-rate heterogeneity and the covariance structure of lifecycle earnings. Journal of Labor Economics, 15(2).

Baker, M. \& Solon, G. (1999). Earnings dynamics and inequality among Canadian men. NBER Working Paper Series, Working Paper 7370.

Barros, R. P., Henriques, R., \& Mendonça, R. (2001). A estabilidade inaceitável: Desigualdade e pobreza no Brasil. Texto para Discussão 800, IPEA.

Cappellari, L. (2000). The dynamics and inequality of Italian male earnings: Permanent changes or transitory fluctuations? ISER Working Paper 2000-41, University of Essex.

Chamberlain, G. (1982). Multivariate regression models for panel data. Journal of Econometrics, 18.

Chamberlain, G. (1984). Panel data. In Grilliches \& Intriligator, editors, Handbook of Econometrics. North-Holland.

Dickens, R. (2000). The evolution of individual male earnings in Great Britain: 1975-95. The Economic Journal, 110.

Fields, G. (2001). Distribution and Development: A New Look at the Developing World. Russel Sage Foundation and MIT Press. 
Freije, S. \& Souza, P. A. (2001). Earnings inequality in Venezuela 1995-1997. XVIII Latin American Meeting of the Econometric Society, http://www.udesa.edu.ar/lames2002/Program1.htm.

Hamilton, J. (1994). Time Series Analysis. Princeton University Press.

Hoffmann, R. (2002). A distribuição de renda no Brasil no período 1992-2001. Economia e Sociedade, 19.

Lillard, L. \& Willis, R. (1978). Dynamic aspects of earnings mobility. Econometrica, 46.

MaCurdy, T. M. (1982). The use of time series processes to model the error structure of earnings in a longitudinal data analysis. Journal of Econometrics, 18.

Menezes Filho, N. A. (2001). Educação e desigualdade. In Menezes Filho, N. A. \& Lisboa, M., editors, Microeconomia e Sociedade no Brasil. Contra Capa.

Moffit, R. \& Gottschalk, P. (1995). Trends in the covariance structure of earnings in the US: 1969-1987. Working Paper 355, Brown University. Fonte: www.econ.jhu/People/Moffitt/mg2_0795.pdf.

Newey, W. (1985). Generalized method of moments specification testing. Journal of Econometrics, 29.

Ramos, X. (2003). The covariance structure of earnings in Great Britain, 19911999. Economica, 70.

Souza, A. P. (2002). Earnings inequality changes in Brazil: Market forces, macroeconomic instability and labor market institutions (1981-1997). Working Paper 02-W15, Vanderbilt University.

Spanos, A. (1986). Statistical Foundations of Econometric Modeling. Cambridge University Press.

Wooldridge, J. (2002). Econometric Analysis of Cross Section and Panel Data. The MIT Press. 


\section{Appendix 1}

\section{Descriptive Statistics}

Table A.1

Log of average real earnings, maximum and minimum values per year

\begin{tabular}{cccc}
\hline & $\begin{array}{c}\text { Log of avg } \\
\text { earnings }\end{array}$ & $\begin{array}{c}\text { Max } \\
\text { log- } \\
\text { earnings }\end{array}$ & $\begin{array}{c}\text { Min log- } \\
\text { earnings }\end{array}$ \\
\hline 1990 & 7.004809 & 9.748496 & 3.758032 \\
1991 & 7.456273 & 9.958274 & 3.972911 \\
1992 & 7.150461 & 9.553937 & 3.562806 \\
1993 & 7.288584 & 9.875608 & 3.890329 \\
1994 & 7.408513 & 9.877222 & 3.887259 \\
1995 & 7.518097 & 10.03233 & 4.045207 \\
1996 & 7.585289 & 10.06238 & 4.071332 \\
1997 & 7.62743 & 10.08905 & 4.097838 \\
1998 & 7.593747 & 10.1439 & 4.153274 \\
\hline
\end{tabular}

Table A.2

Proportion of education levels per year

\begin{tabular}{ccccc}
\hline & $\begin{array}{c}\text { Up to } \\
\text { the 4th } \\
\text { grade }\end{array}$ & $\begin{array}{c}\text { Up to } \\
\text { the 8th } \\
\text { grade }\end{array}$ & $\begin{array}{c}\text { Up to } \\
\text { high } \\
\text { school }\end{array}$ & College \\
\hline 90 & 0.41 & 0.30 & 0.17 & 0.11 \\
91 & 0.41 & 0.30 & 0.18 & 0.11 \\
92 & 0.40 & 0.31 & 0.18 & 0.12 \\
93 & 0.39 & 0.31 & 0.18 & 0.12 \\
94 & 0.38 & 0.31 & 0.19 & 0.12 \\
95 & 0.37 & 0.31 & 0.19 & 0.12 \\
96 & 0.39 & 0.32 & 0.18 & 0.12 \\
97 & 0.36 & 0.32 & 0.19 & 0.13 \\
98 & 0.35 & 0.32 & 0.20 & 0.13 \\
\hline
\end{tabular}

Table A.3

Proportion of age groups per year

\begin{tabular}{ccccc}
\hline & $25-$ & $30-$ & $40-$ & $50-$ \\
& 29 & 39 & 49 & 64 \\
\hline 90 & 0.28 & 0.49 & 0.19 & 0.04 \\
91 & 0.23 & 0.51 & 0.21 & 0.05 \\
92 & 0.17 & 0.52 & 0.24 & 0.06 \\
93 & 0.12 & 0.54 & 0.27 & 0.08 \\
94 & 0.06 & 0.55 & 0.30 & 0.09 \\
95 & 0.00 & 0.56 & 0.33 & 0.11 \\
96 & 0.00 & 0.51 & 0.37 & 0.12 \\
97 & 0.00 & 0.45 & 0.40 & 0.15 \\
98 & 0.00 & 0.40 & 0.43 & 0.17 \\
\hline
\end{tabular}




\section{Appendix 2}

\section{Minimum Distance Methods}

The aim of this section is to describe the methods used to estimate and test earnings autocovariance models. Let $\mathbf{m}$ be a column vector which stacks the elements of the empirical autocovariance matrix of earnings. Since the matrix is symmetric, m contains only the columns of its lower (or upper) triangle, therefore its order is $[t(t+1) / 2] \times 1$. The variance-covariance matrix of the elements in $\mathbf{m}$ can be estimated in the usual fashion (Abowd and Card, 1989):

$$
\hat{\mathbf{V}}=\frac{1}{N} \sum_{i}\left(\mathbf{m}_{i}-\mathbf{m}\right)\left(\mathbf{m}_{i}-\mathbf{m}\right)^{\prime}
$$

where $\mathbf{m}_{i}$ is obtained from the lower (or upper) triangle of $\mathbf{y}_{\mathbf{i}} \mathbf{y}_{\mathbf{i}}^{\prime}$, the cross product matrix for the earnings of each individual $\left(\mathbf{y}_{\mathbf{i}}\right.$ is a vector $t \times 1$ that contains the $t$ earnings of each individual). As $\mathbf{y}_{\mathbf{i}} \mathbf{y}_{\mathbf{i}}^{\prime}$ is also symmetric, its dimension will be $[t(t+1) / 2] \times 1$.

Under relatively general conditions (Abowd and Card (1989); Chamberlain (1982, 1984)), the following property holds:

$$
\sqrt{N}(\mathbf{m}-\boldsymbol{\mu}) \stackrel{D}{\rightarrow} N\left(\mathbf{0}, \mathbf{V}^{*}\right)
$$

where $\boldsymbol{\mu}=E(\mathbf{m})$, i.e., the vector that contains the elements of the true variancecovariance matrix, and $\mathbf{V}^{*}=E\left(\mathbf{m}_{i} \mathbf{m}_{i}^{\prime}\right)-E\left(\mathbf{m}_{i}\right) E\left(\mathbf{m}_{i}^{\prime}\right)$.

Let us define $\boldsymbol{\mu}=\mathbf{g}(\boldsymbol{\theta})$ as a model for the covariance elements, that is, covariances may be written as a known function of the parameters of vector $\boldsymbol{\theta}$, whose order is $q$. In our models, the order of $\mathbf{m}$, denoted by $p$, will always be superior to the order of $\boldsymbol{\theta}$, i.e., we will always have $p>q$, and therefore, the models will always be overidentified. A way to estimate these parameters is to use minimum distance methods (Chamberlain (1982, 1984), Abowd and Card (1989)). This method consists in minimizing the following quadratic form:

$$
\underset{\theta}{\operatorname{Min}}[\mathbf{m}-\mathbf{g}(\boldsymbol{\theta})]^{\prime} A[\mathbf{m}-\mathbf{g}(\boldsymbol{\theta})]
$$

where $\mathbf{A}$ is an appropriate weighting matrix, $\mathbf{A} \stackrel{a . s .}{\rightarrow} \boldsymbol{\Psi}$ and $\boldsymbol{\Psi}$ is a positive-definite matrix (Chamberlain, 1984). In other words, the parameters should be chosen so as to bring the distance between the observed and theoretical second moments as close to zero as possible.

Under certain regularity conditions (Chamberlain, 1984), the following properties are valid for the minimum distance estimator:

1. $\hat{\theta} \stackrel{\text { a.s. }}{\rightarrow} \boldsymbol{\theta}^{*}$, i.e., the estimator converges almost surely to the true value $\boldsymbol{\theta}^{*}$;

2. $\sqrt{N}\left(\hat{\boldsymbol{\theta}}-\boldsymbol{\theta}^{*}\right) \stackrel{D}{\rightarrow} N(0, \boldsymbol{\Lambda})$, where: 


$$
\mathbf{\Lambda}=\left(\mathbf{G}^{\prime} \mathbf{A} \mathbf{G}\right)^{-\mathbf{1}} \mathbf{G}^{\prime} \mathbf{A V A G}\left(\mathbf{G}^{\prime} \mathbf{A G}\right)^{-\mathbf{1}}
$$

where $\mathbf{G}$ is the Jacobian matrix (of order $q \times p$ ) $\partial \mathbf{g}(\boldsymbol{\theta}) / \partial \boldsymbol{\theta}$ assessed at the estimated value for $\boldsymbol{\theta}$. This expression shows that the optimal weighting matrix (as far as efficiency is concerned) is given by $\mathbf{A}=\mathbf{V}^{-\mathbf{1}}$, the inverse of the variance matrix of covariance elements, since in this case $\boldsymbol{\Lambda}$ is reduced to $\left(\mathbf{G}^{\prime} \mathbf{V}^{-\mathbf{1}} \mathbf{G}\right)^{-\mathbf{1}}$ only. This procedure leads to the optimal minimum distance estimator, hereinafter OMD.

However, despite the efficiency of this estimator, Altonji and Segal (1996) amassed evidence from Monte Carlo's method that the OMD estimator is biased in small samples. These authors suggest using identity matrix weighting, a procedure we will refer to as EWMD (equally weighted minimum distance) - which is actually a least squares estimation.

Besides providing estimates for the parameters of interest, the minimum distance method can also be used as a basis for testing the model's general structure. For OMD, the following test statistics is used (Chamberlain (1984); Abowd and Card (1989)):

$$
N\left[\mathbf{m}-\mathbf{g}\left(\boldsymbol{\theta}^{0}\right)\right]^{\prime} \mathbf{V}^{-1}\left[\mathbf{m}-\mathbf{g}\left(\boldsymbol{\theta}^{0}\right)\right]
$$

The minimized quadratic form multiplied by $N$ (number of individuals in each cross-section of the balanced panel) is asymptotically distributed as chi-square with degrees of freedom equal to the dimension of $\mathbf{m}$ minus the rank of the Jacobian matrix $\partial \mathbf{g}(\theta) / \partial \boldsymbol{\theta}$ assessed at the true value of $\boldsymbol{\theta}$. The null hypothesis that the restriction $\mathbf{m}=\mathbf{g}(\boldsymbol{\theta})$ is correct is equivalent to stating that the structure imposed by the model is valid.

In the general case, for a quadratic form weighted by a positive-definite matrix A, we have the following test expression (Newey (1985), Abowd and Card (1989), Dickens (2000)):

$$
N\left[\mathbf{m}-\mathbf{g}\left(\boldsymbol{\theta}^{A}\right)\right]^{\prime} \mathbf{R}^{-}\left[\mathbf{m}-\mathbf{g}\left(\boldsymbol{\theta}^{A}\right)\right]
$$

where $\mathbf{R}^{-}$is a generalized inverse of $\mathbf{R}=\mathbf{W} \mathbf{V} \mathbf{W}^{\prime}$,

$\mathbf{W}=\mathbf{I}-\mathbf{G}\left(\mathbf{G}^{\prime} \mathbf{A} \mathbf{G}\right)^{-\mathbf{1}} \mathbf{G}^{\prime} \mathbf{A}$, and $\mathbf{G}$ is the Jacobian matrix (of order $q \times p$ ) $\partial \mathbf{g}(\boldsymbol{\theta}) / \partial \boldsymbol{\theta}$ assessed at $\boldsymbol{\theta}^{A}$, the estimator obtained from the method. This statistics also follows the asymptotic chi-square distribution. This is the statistics used for EWMD, in which $\mathbf{A}=\mathbf{I}$.

Moreover, besides being used as a test for the model's general structure, this chi-square statistic can also be considered as a goodness-of-fit test; therefore, it is also useful for comparison across models.

In addition to these tests for the model's general structure, it is also possible to obtain standard deviations for $t$-tests on individual coefficients. As seen, $\hat{\boldsymbol{\theta}}$ is $\sqrt{N}$ asymptotically normally distributed and $\boldsymbol{\Lambda}$ is the asymptotic variance of $\sqrt{N}(\hat{\boldsymbol{\theta}}-$ $\left.\boldsymbol{\theta}^{*}\right)$. Even if $\boldsymbol{\Lambda} / N=\operatorname{Var}(\hat{\boldsymbol{\theta}})$ is valid only in special cases and although $\hat{\boldsymbol{\theta}}$ seldom 
has an accurate normal distribution, in practice, $\hat{\boldsymbol{\theta}}$ is treated as if (Wooldridge, 2002):

$$
\hat{\boldsymbol{\theta}} \sim N(\boldsymbol{\theta}, \boldsymbol{\Lambda} / N)
$$

Thus, we can estimate the variance of $\hat{\boldsymbol{\theta}}$ using the following expression:

$$
\operatorname{Avâr}(\hat{\boldsymbol{\theta}})=\left[\left(\mathbf{G}^{\prime} \mathbf{A} \mathbf{G}\right)^{-\mathbf{1}} \mathbf{G}^{\prime} \mathbf{A} \hat{\mathbf{V}} \mathbf{A G}\left(\mathbf{G}^{\prime} \mathbf{A G}\right)^{-\mathbf{1}}\right] / N
$$

